\title{
Análisis cefalométrico de tejidos blandos de Trujillo aplicado a planeación virtual
}

\author{
Trujillo's soft tissue cephalometric analysis applied to virtual planning
}

Juan José Trujillo Fandiño,* Oscar Saavedra Arias

\section{RESUMEN}

Actualmente la tecnología se encuentra presente en todos los ámbitos de nuestras vidas, desde los más sencillos hasta los más complejos, desde los presentes en nuestro modo de vida hasta los presentes en nuestras actividades laborales. La inclusión de estas tecnologías se debe a las ventajas que éstas ofrecen al momento de realizar nuestras tareas y por lo general son tecnologías que se aplican a métodos ya establecidos, por ejemplo; bicicleta, carreta tirada por caballo, carro o radio, televisión blanco y negro, televisión a color. Los principios fundamentales son los mismos, únicamente la tecnología actual mejora la forma en la que podemos aplicar estos fundamentos. Del mismo modo en el área de la cirugía ortognática ha surgido una tecnología en la que muchos creen y sostienen que llegó para quedarse, es la tecnología de la realidad virtual, desde este enfoque el equipo de trabajo que presenta este artículo, tomo el reconocido y muy funcional análisis cefalométrico de tejidos duros del Dr. Juan José Trujillo Fandiño (análisis de Trujillo) y lo adapto para poder realizar las mediciones de dicho análisis en tecnología tridimensional. Conclusiones: El análisis de tejidos duros de Trujillo cuenta con mediciones de todas las estructuras óseas involucradas en la cirugía ortognática, por lo que nos parece el análisis indispensa-

\section{ABSTRACT}

Today technology is present in all areas of our lives, from the simplest to the most complex, from those present in our way of life to those present in our work activities. The inclusion of these technologies is due to the advantages they offer when performing our tasks and they are usually technologies that are applied to already established methods, for example; bicycle, horse-drawn cart, car or radio, black and white television, color television. The fundamental principles are the same only today's technology improves the way we can apply these fundamentals. Similarly in the area of orthognathic surgery has emerged a technology that many believe and argue that came to stay, is the technology of virtual reality, from this approach the team that presents this article, I take the recognized and very functional cephalometric analysis of hard tissues of Dr. Juan José Trujillo Fandiño (Trujillo's analysis) and adapt it to make the measurements of such analysis in three-dimensional technology. Conclusions: Trujillo's hard tissue analysis has measurements of all the bone structures involved in orthognathic surgery, so it seems to us to be the indispensable analysis for the diagnostic complement of the clinical examination, indispensable as pre-surgical cephalometric analysis.

* Médico adscrito al Hospital Juárez de México.

‡ Servicio de Cirugía Maxilofacial del Nuevo Hospital Civil de Guadalajara «Dr. Juan I. Menchaca». Guadalajara, Jalisco. 
ble para el complemento diagnóstico al examen clínico, indispensable como análisis cefalométrico prequirúrgico.

Palabras clave: Cefalometría, cirujanos orales y maxilofaciales, cirugía ortognática, ortodoncia.

\section{INTRODUCCIÓN}

La cefalometría 2D (dos dimensiones) fue introducida en 1931 mediante la cefalografía, ${ }^{1}$ basados en esta tecnología la cefalometría convencional ha sido una de las herramientas diagnósticas estándar para el análisis de las deformidades maxilofaciales, problemas ortodónticos, valorar el crecimiento y evaluación de los cambios luego de un tratamiento. ${ }^{2}$

En síntesis, la cefalometría 2D se utiliza para hacer mediciones de forma, tamaño, posición y orientación de las diferentes unidades de la cara. ${ }^{3}$ Pero los cefalogramas tienen limitaciones por su naturaleza de ser proyecciones en dos dimensiones de estructuras de tres dimensiones; por lo que carecen de perspectiva, presentan errores de proyección, distorsión de la geometría, variaciones de magnificación, errores de la posición de la cabeza. ${ }^{4}$

La tecnología de las imágenes obtenidas mediante tomografía computada helicoidal y la tomografía computada de haz de Cono, han desencadenado una revolución tecnológica que permitió el desarrollo de la tecnología 3D aplicada a la región craneofacial, que nos ha provisto de representaciones reales de la anatomía del paciente. En ciertos pacientes que presentan anomalías craneofaciales, hendiduras orofaciales, asimetrías craneofaciales, los cefalogramas convencionales no son en la actualidad una herramienta diagnostica óptima. ${ }^{5}$

La transición de los métodos basados en dos dimensiones (2D) a la aplicación de los métodos en tres dimensiones (3D) deben ser meticulosamente evaluados, deben cumplir con normas de calidad, tener principios científicos y deben ser capaces de someterse al método científico para su validez. De lo contrario, su funcionalidad quedará en duda y no podrá ser aplicada a la práctica clínica.

En la literatura hay más de una década en el que se han aplicado protocolos científicos aplicados a la comparación de la precisión entre la cefalometría $2 \mathrm{D}$ y $3 \mathrm{D} .^{2,6-10}$ Las conclusiones son diversas, pero en resumen la evidencia menciona dos problemas fundamentales con la cefalometría 2D al compararla con la cefalometría 3D; el primero es que muchos
Keywords: Cephalometry, oral and maxillofacial surgeons, orthognathic surgery, orthodontics. parámetros importantes no pueden ser medidos. El segundo es que la mayoría de las mediciones cefalométricos en $2 \mathrm{D}$ están distorsionadas en presencia de asimetrías faciales.

Por lo tanto, si la evidencia científica muestra que existe mayor precisión en la tecnología tridimensional, es conveniente iniciar con la transición de los protocolos de nuestros métodos bidimensionales a protocolos tridimensionales, que tengan la capacidad de ser reproducibles y que sus resultados puedan ser medidos.

El objetivo de este artículo es presentar la adaptación del conocido análisis cefalométrico del Dr. Trujillo a un análisis cefalométrico tridimensional.

\section{BASES TEÓRICAS DE LA CEFALOMETRÍA TRIDIMENSIONAL (3D)}

El análisis cefalométrico idóneo debe tener la capacidad de medir cinco atributos geométricos de cada subunidad de la cara. ${ }^{11}$ Estos cinco atributos son: tamaño, posición, orientación, forma y simetría. Tres de éstos (posición, orientación y simetría) requieren de un marco de referencia. ${ }^{2}$

Para la cefalometría bidimensional se utiliza un sistema de «geometría analítica plana» denominado: «sistema diédrico ortogonal» 0 «sistema coordenado cartesiano» el nombre cartesiano es en honor del filósofo y matemático René Descartes (1596-1650). Este sistema está constituido por dos rectas perpendiculares que se intersecan en un punto «O» (el origen). Una de las rectas se representa en posición horizontal y se le da el nombre de eje $x$. A la otra recta se le representa vertical y se le denomina eje y. Ambas constituyen los dos ejes de coordenadas rectangulares de un plano (Figura 1). ${ }^{12}$

Así pues, en la geometría analítica plana solamente se consideran los puntos situados en un solo plano (plano sagital en el análisis cefalométrico lateral bidimensional).

Esta limitación no permite la investigación de las subunidades de la cara en el espacio.

Con el fin de extender el método analítico al estudio de las subunidades de la cara en tres dimensiones, aplicamos «la geometría analítica del espacio». La 
geometría analítica del espacio requiere del «sistema triédrico ortogonal» 0 "sistema de coordenadas rectangulares en el espacio». Donde se consideran tres planos mutuamente perpendiculares que se cortan en el punto común «O», como los objetos en estudio van a localizarse con referencia a estos elementos, los planos se llaman «planos coordenados», las rectas de intersección de estos planos se denominan «ejes coordenados» y el punto $\mathrm{O}$ «origen del sistema de coordenadas rectangular» (Figura 2). ${ }^{13}$

Este sistema de coordenadas se convierte en nuestro marco de referencia global, el cual abarca toda la cabeza y sirve para orientar la cabeza (Figuras 3 y 4 ).

Se utilizan marcos de referencia locales, los marcos de referencia local son para las orientaciones de las subunidades de la cara (maxilar, mandíbula, mentón, incisivo superior, etc.). Sirven para deducir la orientación de las subunidades de la cara con respecto al marco global (Figuras 5 y 6 ).

Para entender este concepto hay que visualizar una iglesia, la cual está perfectamente orientada; el techo está arriba, el piso está abajo y las paredes están alineadas con el norte, sur, este y oeste. El púlpito que está en un extremo de la iglesia puede estar orientado hacia una esquina de la iglesia, por lo tanto, su parte superior, inferior y sus lados tienen una alineación independiente, por lo que se denomina «sistema de coordenadas local», al conocer el sistema de coordenadas global, automáticamente con geometría analítica y trigonometría podemos deducir la posición espacial y la orientación del púlpito, de las bancas de la iglesia, etc.

\section{MARCO TEÓRICO DE MEDICIONES 3D}

La mayoría de las mediciones cefalométricas son ángulos y distancias (ángulos entre; 2 líneas, 1 línea y 1 plano, 2 planos), (distancias entre; 2 puntos, 1 punto y 1 línea, 1 punto y un plano).

En el sistema coordenado de dos dimensiones un punto está definido por un valor en; $x$ (eje horizontal), y (eje vertical) por ejemplo un punto es: punto $1=$ $\mathrm{P} 1(3,6)$ donde 3 representa a $X$ y 6 representa a Y (Figura 1).

En el sistema coordenado de dos dimensiones una distancia entre dos puntos se calcula con la siguiente fórmula:

$$
d=\sqrt{ }\left(x_{2}-x_{1}\right)^{2}+\left(y_{z}-y_{1}\right)^{2}
$$

Donde las coordenadas del punto 1 son; P1 (X1, Y1) y las coordenadas del punto 2 son; P2 (X2, Y2).
Pero en mediciones 3D se adiciona un nuevo eje de coordenada, el eje z (vertical) (Figura 2) y la fórmula de distancia entonces es:

$$
d=\sqrt{ }\left(x_{2}-x_{1}\right)^{2}+\left(y_{2}-y_{1}\right)^{2}+\left(z_{2}-z_{1}\right)^{2}
$$

Y aunque en teoría solo se agrega un valor por cada punto, en la práctica del análisis cefalométrico $3 \mathrm{D}$ esto tiene implicaciones que debemos analizar para realizar mediciones adecuadas.

También hay ecuaciones matemáticas relacionadas con las mediciones de los ángulos:

$$
\theta=\operatorname{acos}([a x b] /|a| x|b|)
$$

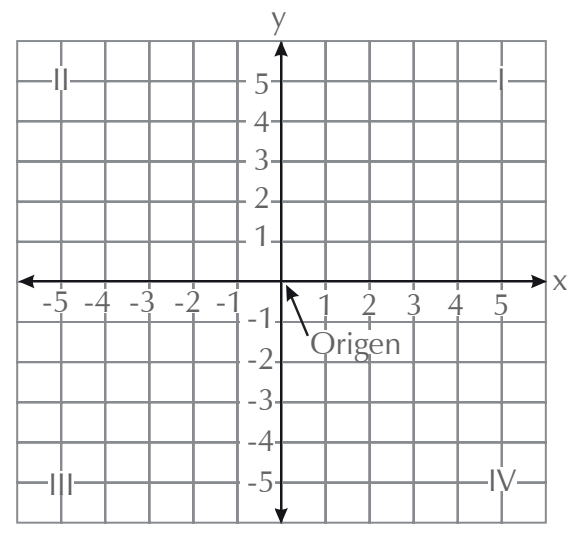

Figura 1: Eje x horizontal, eje y vertical. Este plano cartesiano forma cuatro cuadrantes determinando los datos de «X» $\mathrm{y}$ de «y» podemos posicionar un punto.

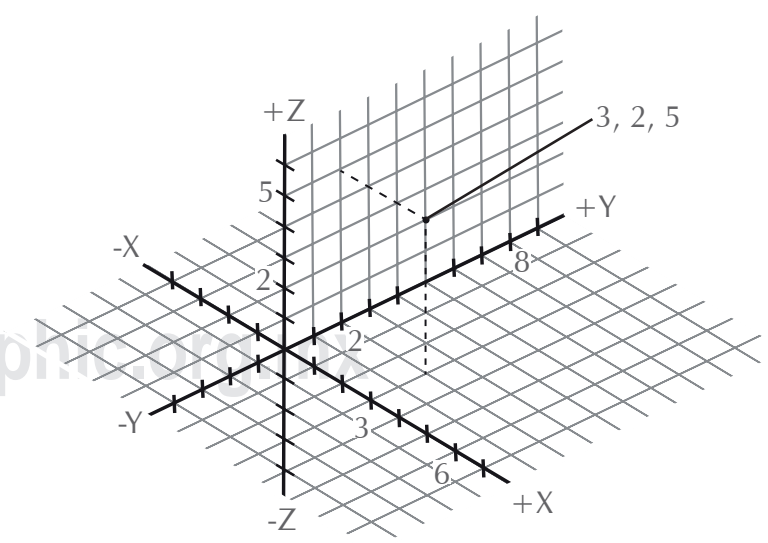

Figura 2: Sistema cartesiano de coordenadas rectangulares. Se observa eje $z$ vertical, eje $x$ horizontal medio lateral, eje $y$ horizontal antero posterior. Estos ejes forman ocho cuadrantes. Se observa un punto con las coordenadas 3, 2, 5 que representan al eje $x, y, z$ respectivamente. 


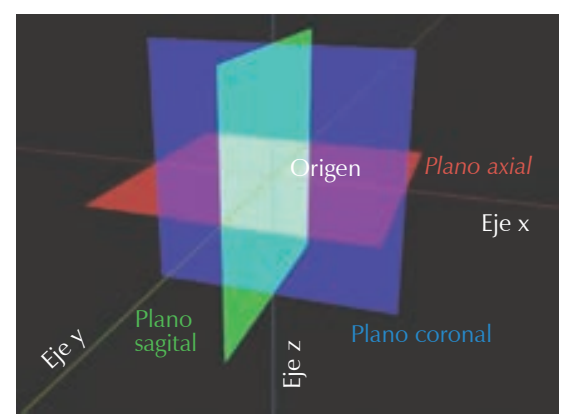

Figura 3: Marco de referencia global. Rojo eje «X» horizontal con plano axial. Verde eje «y» horizontal con plano sagital. Azul eje «z» vertical con plano coronal. Estos ejes y planos son perpendiculares uno a otro y están presentes en todos los softwares que utilizan las tres dimensiones.

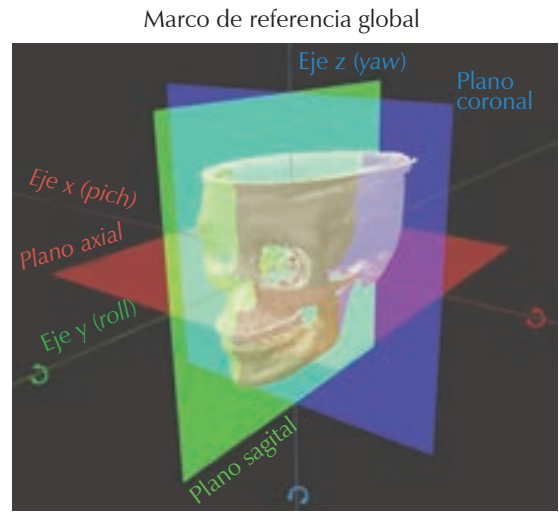

Figura 4: Orientación de cabeza con respecto al marco de referencia global. La rotación del eje «X» se denomina «pich» (cabeceo). La rotación del eje «yy» se denomina «roll» (alabeo). La rotación del eje «z» se denomina «yaw» (guiñada).
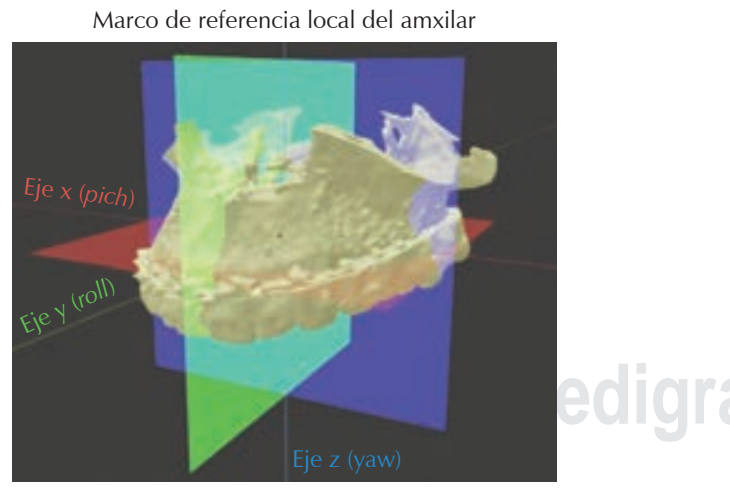

Figura 5: Marco de referencia local del maxilar superior. Tenemos los mismos ejes con sus respectivos planos. Las rotaciones de sus ejes (pich, roll, yaw) es posible al comparar sus angulaciones con respecto al marco de referencia global. Es decir, el pich del marco de referencia global es $0^{\circ}$, pero el plano oclusal del maxilar puede estar inclinado, al medir esa inclinación podemos decir que el maxilar tiene un pich de $13^{\circ}$ por ejemplo.
Marco de referencia global Marco de referencia local de incisivo superior

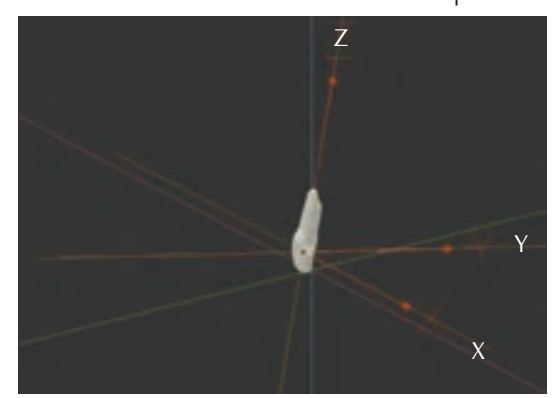

Figura 6: Marco de referencia local del incisivo superior con marco de referencia global. Línea roja eje x (pich: $0^{\circ}$ ), línea verde eje y $\left(\right.$ roll: $\left.0^{\circ}\right)$, línea azul eje z $\left(y a w: 0^{\circ}\right)$. En líneas naranjas y con letra se presentan los ejes del incisivo. Se observa la diferencia de angulación (rotación) entre el marco de referencia global con el local. Midiendo esas rotaciones podemos determinar el (pich, roll y yaw) del incisivo superior con respecto al marco de referencia global.

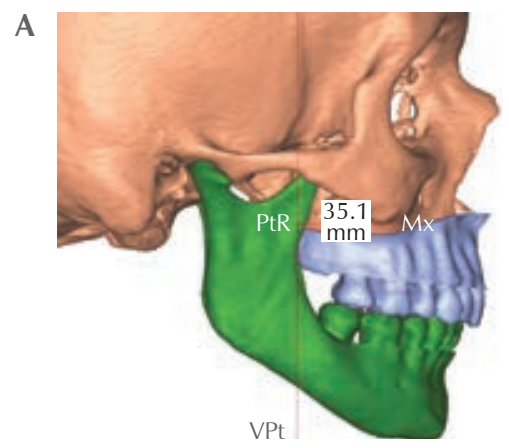

Figura 7: A) Aquí se observa el punto Mx (punto medio entre espina nasal anterior y espina nasal posterior) y el punto PtR (punto pterigoideo derecho). Se programa al software para que haga la medición entre el punto $M x$ y el plano vertical que pasa por el punto PtR (VPt). Se observa una medición de 35.1.

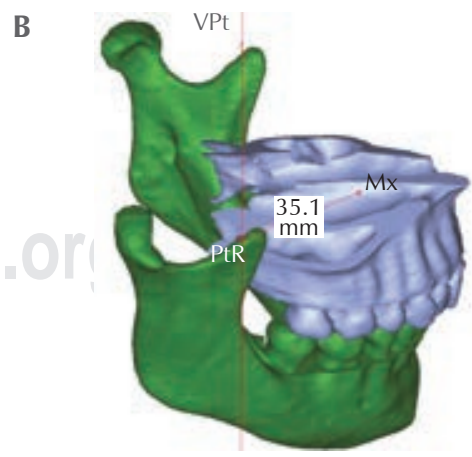

Figura 7: B) Se observa como la medición, aunque es en línea recta en el plano sagital, presenta una pendiente en el plano axial, por lo tanto, la medida es incorrecta. Por esta razón en cefalometría 3D debemos de tener en cuenta los tres ejes y sus respectivos planos. 


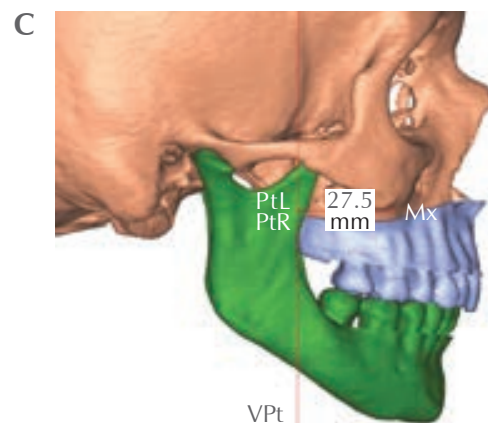

Figura 7: C) VPt (plano vertical pterigoideo). Mx (punto posterior). Aquí generamos un plano el cual pasa por los dos puntos PtR y PtL (punto pterigoideo derecho e izquierdo respectivamente), y es paralelo al plano coronal y perpendicular al eje axial del marco de referencia global.

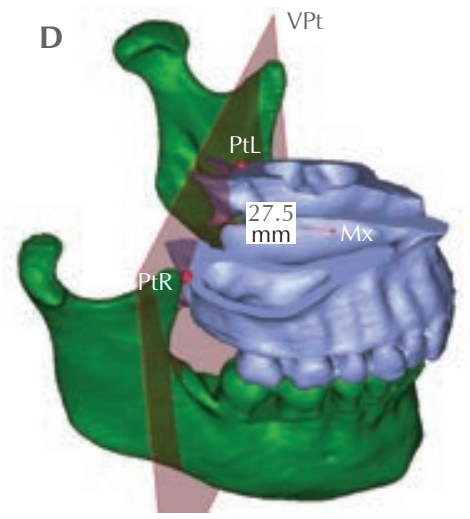

Figura 7: D) Se observa la tridimensionalidad del plano vertical pterigoideo. Medición en línea en el plano sagital y plano axial. Éste es un ejemplo de cómo las mediciones lineales en $3 \mathrm{D}$ se deben realizar entre un punto cefalométrico determinado, y un punto determinado de un plano, dependiendo de las mediciones que queremos realizar.

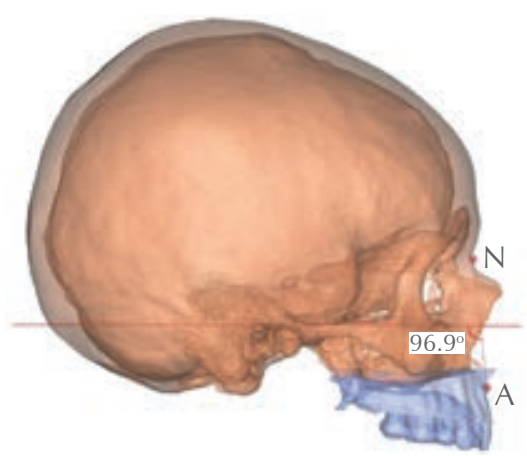

Figura 9: Ejemplo de medida NA-FH, en este caso de $96.9^{\circ}$. Autor: Ricketts. Norma clínica: $90^{\circ} \pm 3^{\circ}$. Aumento: protrusión o prognatismo maxilar. Disminuido: retrusión o prognatismo maxilar.

$\mathrm{N}=$ nación, $\mathrm{A}=$ punto $\langle\mathrm{A} »$.

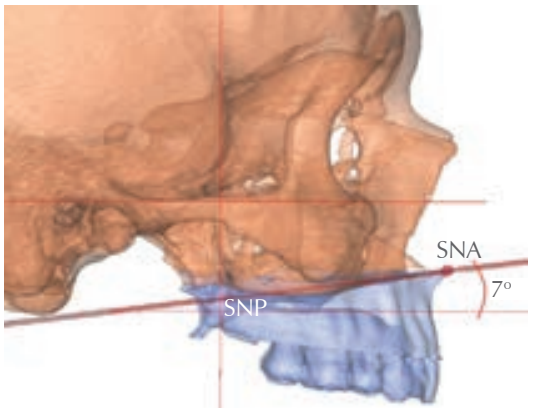

Figura 10: Ejemplo de medida $\mathrm{PMx}-\mathrm{FH}$, en este caso de $7^{\circ}$. Autor: Ricketts. Norma clínica: $-1^{\circ} \pm 3.5^{\circ}$. Aumento: postero-rotación del maxilar o retroinclinación. Disminuido: antero-rotación del maxilar o proinclinación.

$\mathrm{SNA}=$ espina nasal anterior, SNP = espina nasal posterior.

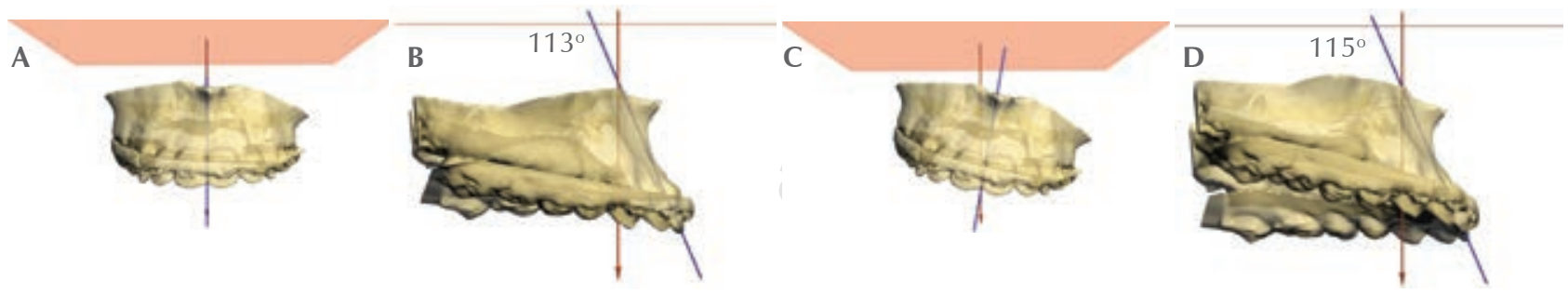

Figura 8: Ejemplo ilustrativo de cómo es medido el ángulo entre línea-plano. A) La línea azul indica el eje del incisivo superior. En naranja se representa el plano de Frankfurt. La rotación del eje «X» (pich) del incisivo superior con respecto al plano de $\mathrm{FH}$ fue de $113^{\circ}$, la rotación fue de $0^{\circ}$ del eje «Y» y «Z» (roll, yaw respectivamente). B) Vista lateral. El ángulo en 3-dimensiones entre el incisivo y el plano de $\mathrm{FH}$ fue de $113^{\circ}$. C) Se observa la rotación de $10^{\circ}$ en sentido de las agujas del reloj en el eje «Y» (roll), el eje «X» (pich) se mantuvo en $113^{\circ}$, el eje «Z» (yaw) se mantuvo en $0^{\circ}$. Con estas nuevas medidas se realizó la misma medición y en este caso el ángulo entre el incisivo y el plano $\mathrm{FH}$ fue de $115^{\circ}, 2^{\circ}$ grados mayor a la medición anterior. D) vista lateral. Tomado de: Gateno J et al. ${ }^{6}$ 
Donde $a$ y $b$ representan los vectores de los planos o las líneas.

Y aunque el tema de las ecuaciones podría ser de interés para algunos, para la mayoría de los cirujanos, la cuestión importante es cómo estas mediciones 3D pueden afectar sus decisiones clínicas. Por esta razón no profundizaremos en el tema de las ecuaciones.

Para ejemplificar algunas de las cuestiones que debemos tener en cuenta para que nuestras mediciones sean correctas, hablaremos de las distancias entre dos puntos, entendiendo que una línea recta, por definición es el segmento de una sucesión de puntos en el espacio, entonces una medida cefalométrica entre un punto y una línea se convierte en la distancia entre un punto cefalométrico determinado y un punto de la sucesión de puntos que forman esa línea, en cefalometría 2D no tienen mayor relevancia este aspecto, pero el ejemplo de las Figuras $7 A-D$ se observa cómo se deben hacer las mediciones. En este ejemplo medimos la distancia entre el punto Mx (maxilar: punto equidistante entre la espina nasal anterior y la espina nasal posterior) y la vertical pterigomaxilar (Mx-VPt) del autor Trujillo. En cefalometría 2D generamos una vertical que pase por el punto VPt (el punto más inferior de la fisura pterigomaxilar) y medimos la distancia entre el punto $\mathrm{Mx}$ y un punto de esta vertical en línea recta horizontal (Figuras $7 \mathrm{~A}$ y B). En cefalometría 3D tenemos que contemplar el otro eje, ya que las mediciones no sólo pueden ser anteroposteriores o superoinferiores, sino que también mediolaterales. Esto nos estrega mayor precisión a la hora de recolectar datos de los estudios de imagen, pero debemos entender los principios para asegurar que los datos recolectados son los reales, en las Figuras $7 C$ y $D$ observamos cómo una distancia desde el punto $\mathrm{Mx}$ a un punto central en un plano vertical que pase a través del punto $\mathrm{Pt}$ derecho y Pt izquierdo.

En un artículo publicado en el 2011 por Gateno y col. abordan el tema de la medición de ángulos entre líneas y planos. Realizan un experimento el cual consiste en medir la inclinación del incisivo superior central con respecto al plano de Frankfurt. Los ángulos línea-plano en 3D se calculan primero determinando el vector normal del plano y luego utilizando la ecuación de ángulo para dos líneas.

En la Figura 8, tomada del artículo de Gaateno, ${ }^{14,15}$ se calcula el ángulo entre el eje largo del incisivo y el plano de Frankfurt, en este ejercicio el incisivo central está orientado con un eje x (pich: cabeceo) de $113^{\circ}$, el eje y (roll: alabeo) de $0^{\circ}$, y el eje z (yaw: guiñada) de $0^{\circ}$. Como era esperado, el ángulo $3 \mathrm{D}$ entre el incisivo y el $\mathrm{FH}$ fue de $113^{\circ}$ (Figuras $8 A$ y B). Posteriormente se giró el incisivo central superior alrededor de un eje anteroposterior o eje y (roll) $10^{\circ}$, el eje $x$ (pich) se mantuvo en $113^{\circ}$ y el eje $z$ (yaw) se mantuvo en $0^{\circ}$. Esta transformación cambió la medición 3D del ángulo entre el incisivo superior y el plano de Frankfurt a $115^{\circ}, 2^{\circ}$ grados más abierto que la medición anterior (Figuras 8 C y D). Según los autores este experimento se observa las diferencias entre las mediciones 2D convencionales y las mediciones 3D. En las mediciones 2D siempre interpretamos el ángulo entre el incisivo y $\mathrm{FH}$ como el «pich» (cabeceo) del incisivo en relación con la horizontal de Frankfurt. $Y$ en las mediciones $3 D$ este ángulo es una combinación de los ejes $\mathrm{x}, \mathrm{y}, \mathrm{z}$ «pich, roll, yaw». ${ }^{15}$

\section{POSICIÓN NATURAL DE LA CABEZA}

En la aplicación clínica los planos x, y, z, se convierten en los planos axial, coronal y sagital respectivamente. Existen dos métodos informados en la literatura para poder orientar toda la cabeza a estos planos: el primer método consiste en identificar puntos anatómicos clave, el segundo consiste en colocar la tomografía en "posición natural de la cabeza». 3,16,17

Nos centraremos a continuación en la orientación conforme al método de (NHP) posición natural de cabeza por sus siglas en inglés.

Una de las primeras referencias encontradas en la literatura (1958) sobre este tema ${ }^{18}$ es un estudio hecho con la hipótesis que la posición natural de la cabeza es relativamente constante, sugieren que de ser cierta esta hipótesis sería posible introducir una línea de referencia extracraneal que se extrapolaría al estudio cefalométrico y tendría la certeza de ser una vertical verdadera. En resumen, el artículo explica que colocan a pacientes sentados en posición cómoda viendo hacia la imagen de sus ojos reflejados en un espejo colocado a nivel de sus pupilas a 1.70 metros de distancia. Colocaban una plomada vertical y con esta configuración tomaban la radiografía. De tal modo conseguían una radiografía lateral de cráneo con una «vertical verdadera».

Actualmente hay tres protocolos útiles para transferir y orientar el estudio tomográfico a una posición natural de la cabeza (NPH) en el software y alinearlo con los planos axial, coronal y sagital.

El primer método consiste en tomar fotografías laterales y frontales mientras el paciente está en 
una posición natural de la cabeza. Esta fotografía requiere de una calibración de la cámara para que su plano focal sea perpendicular al suelo, además requiere que en el fondo de la fotografía haya una plomada para tener una «vertical verdadera». Ya con las fotografías orientamos de manera manual la tomografía con las fotografías. ${ }^{19}$

El segundo método consiste en colocar al paciente en (NPH) y utilizar un láser autonivelante que proyecta una línea vertical y una horizontal sobre la eminencia malar, se coloca un marcador radiopaco sobre estas líneas, el paciente se somete al estudio tomográfico y estos marcadores sirven para orientar la cabeza con el plano sagital y axial respectivamente. ${ }^{20}$

El tercer método consiste en la orientación de la tomografía con un sensor digital de orientación. Consiste en un giroscopio digital que está unido a una plantilla de mordida, se pide al paciente que muerda la plantilla, se coloque en (NPH) y el giroscopio digital registra la orientación de los eje axial, coronal y sagital, con estos datos se orienta la tomografía a la NHP del paciente. ${ }^{21}$

Estos métodos están basados en la hipótesis de que la NHP es una postura reproducible, es decir que no importa quién solicite al paciente que adopte esa postura y no importa cuántas veces en diferentes momentos se le solicite, siempre será la misma postura. Pero en la literatura hay estudios que reportan que hay un margen de error de $2^{\circ}$ en el eje axial, lo cual en cefalometría bidimensional no representa un error clínicamente significativo, pero no hay estudios sobre la reproducibilidad con respecto a los ejes coronal y sagital.

Suponiendo que hay también hay un margen de error de $2^{\circ}$ en el eje sagital, entendiendo que la mirada al horizonte es la forma de solicitar al paciente que adopte el NHP, el eje sagital pasa entre la línea bipupilar entre los huesos nasales, una discrepancia de $2^{\circ}$, haría que los datos recolectados de la posición de la línea media inter-incisal superior y pogonión varíen 2.8 y $4.2 \mathrm{~mm}$ respectivamente, asumiendo que la distancia entre nasión y los incisivos superiores es de $80 \mathrm{~mm}$ y de $120 \mathrm{~mm}$ hasta pogonión. 3,19

Por lo anterior en casos de tratar a pacientes sin síndromes o asimetrías de tercio medio, utilizamos el protocolo publicado en la Revista de Cirugía CráneoMaxilofacial (2017) por Heufelder y colaboradores ${ }^{17}$ el cual consiste en toma del estudio tomográfico, y se orienta de la siguiente manera: el eje axial con el plano de Frankfurt, el eje coronal con el plano bipupilar, y el eje sagital con el plano de la línea media (es un plano que pasa por tres estructuras de la línea media de la base de cráneo y es perpendicular a los otros 2 planos).

\section{ANÁLISIS 3D DE TEJIDOS DUROS}

Teniendo en cuenta este resumido marco teórico, las modificaciones a continuación mostradas del análisis cefalométrico de tejidos duros de Trujillo, ${ }^{22}$ tuvieron el objetivo final de darle precisión a los datos obtenidos de los estudios radiográficos. La mayoría de las mediciones se realizaron programando el software para medir distancias entre puntos y planos, y la mayoría de los ángulos son mediciones entre dos planos. El software sólo entrega los datos obtenidos y los autores recolectan los datos y los interpretan.

Esta cefalometría se realizó mediante el software Proplan CMF 3.0 de la empresa belga Materialise.

1. NA-FH: proyección del maxilar; es el ángulo formado por la intersección de las líneas nasiónpunto «A» y el plano de Frankfurt o plano horizontal (Figura 9).

2. PMx-FH: inclinación maxilar; es el ángulo formado por el plano maxilar (espina nasal anterior y posterior) y el plano de Frankfurt o plano horizontal (Figura 10).

3. Mx-VPt: posición media maxilar anteroposterior; distancia entre el punto maxilar (Mx: punto equidistante entre la espina nasal anterior y la espina nasal posterior) y el plano de la vertical pterigoidea (Figura 11).

4. Mx-FH: posición media maxilar superoinferior; distancia entre el punto maxilar (Mx: punto equidistante entre la espina nasal anterior y la espina nasal posterior) y el plano horizontal de Frankfurt (Figura 11).

5. ENA-ENP: dimensión maxilar anteroposterior; distancia entre espina nasal anterior y espina nasal posterior (Figura 12).

6. 1-PMx: angulación del incisivo superior con respecto al plano maxilar; medición del ángulo formado por el eje axial del incisivo superior (1) con el plano maxilar (PMx) (Figura 13).

7. 1-FH: angulación del incisivo superior con respecto al plano de Frankfurt; medida del ángulo formado por el eje axial del incisivo superior y el plano horizontal de Frankfurt (Figura 14).

8. 1-NA: angulación clínica del incisivo superior; medición del ángulo formado por el eje axial del incisivo superior y la línea NA (nasión-punto A) (Figuras 15A y B). 


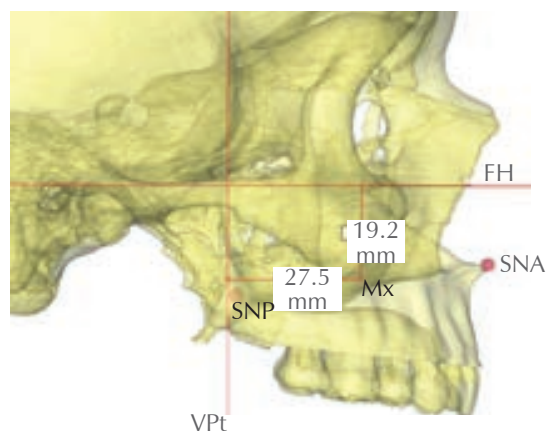

Figura 11: Ejemplo de medida Mx-VPt, en este caso de $27.5 \mathrm{~mm}$. Autor: Trujillo. Norma clínica: mujeres $26 \mathrm{~mm} /$ hombres $30 \mathrm{~mm}( \pm 3 \mathrm{~mm})$. Aumento: protrusión maxilar. Disminuido: retrusión maxilar. Ejemplo de medida de Mx$\mathrm{FH}$, en este caso de $19.2 \mathrm{~mm}$. Autor: Trujillo. Norma clínica: mujeres $27 \mathrm{~mm} /$ hombres $30 \mathrm{~mm}$ ( $\pm 3 \mathrm{~mm}$ ). Aumento: ínfero-posición de la porción media del maxilar. Disminuido: supero-posición de la porción media del maxilar.

$\mathrm{FH}=$ plano de Frankfurt, VPt $=$ plano vertical pterigoideo, SNP $=$ espina nasal posterior, $\mathrm{SNA}=$ espina nasal anterior, $\mathrm{Mx}=$ punto equidistante entre espina nasal anterior y posterior.

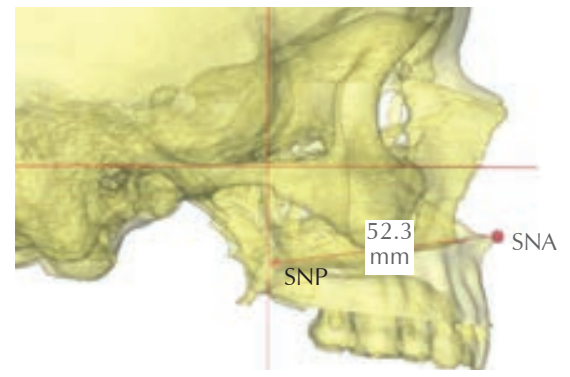

Figura 12: Ejemplo de medida ENA-ENP, en este caso de $52.3 \mathrm{~mm}$. Autor: Trujillo. Norma clínica: mujeres $52 \mathrm{~mm} /$ hombres $59 \mathrm{~mm}( \pm 3 \mathrm{~mm})$. Aumento: macrognatismo maxilar. Disminuido: micrognatismo maxilar. $\mathrm{SNA}=$ espina nasal anterior, SNP = espina nasal posterior.

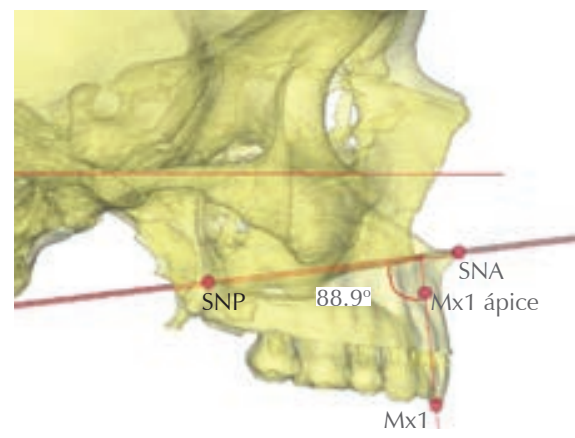

Figura 13: Ejemplo de medida 1-PMx, en este caso de $88.9^{\circ}$. Autor: Trujillo. Norma clínica: $109^{\circ} \pm 3^{\circ}$. Aumento: labioversión de dientes anteriores superiores. Disminuido: palatoversión de dientes anteriores superiores.

$\mathrm{SNA}=$ espina nasal anterior, $\mathrm{SNP}=$ espina nasal posterior, $\mathrm{Mx} 1=$ borde incisal de incisivo superior, $M \times 1$ ápice $=$ ápice de incisivo sup.

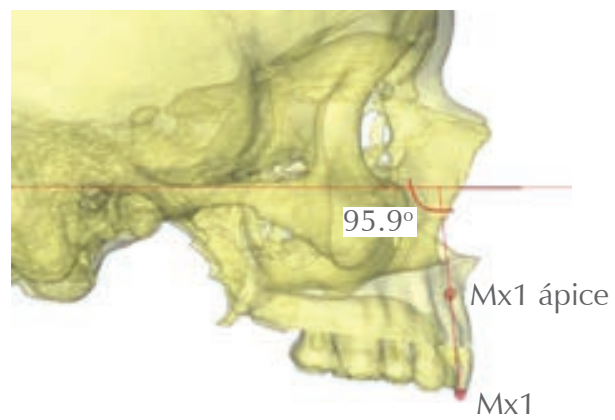

Figura 14: Ejemplo de medida 1-FH, en este caso de 95.9. Autor: Trujillo. Norma clínica: $110^{\circ} \pm 3^{\circ+}$. Aumento: labioversión dentoalveolar anterior superior. Disminuido: palatoversión dentoalveolar anterior superior.

Mx1 = borde incisal de incisivo superior, $M \times 1$ ápice = ápice de incisivo superior.

A

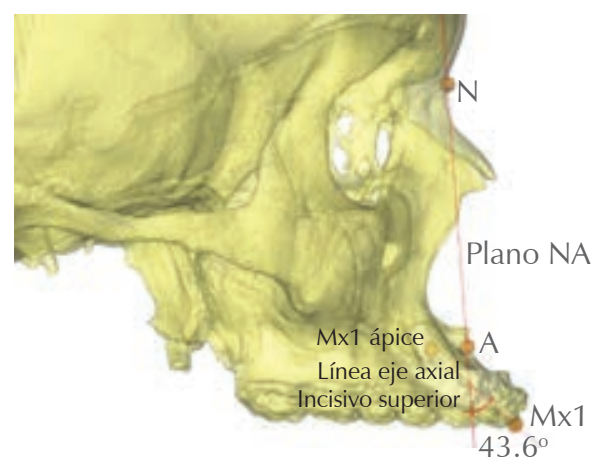

B

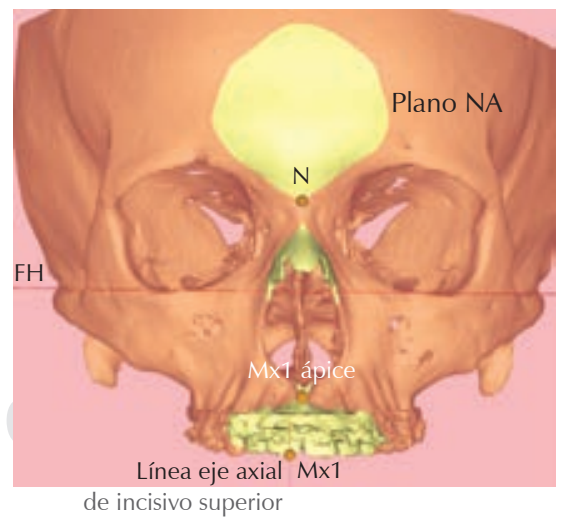

Figura 15: A) Ejemplo de medida 1-NA vista lateral, en este caso de $43.6^{\circ}$. Autor: Steiner. Norma clínica: 22\%/4 mm ( \pm $3^{\circ}$ ). Aumento: labioversión clínica. Disminuido: palatoversión clínica. B) Ejemplo de medida 1-NA vista frontal. Autor: Steiner. En éste se observa al plano NA y su relación con la línea del eje axial del incisivo superior. $\mathrm{N}=$ nasión, $\mathrm{A}=$ punto más cóncavo de maxilar. 
9. 6d-VPt: posición mesiodistal de los primeros molares superiores; distancia del punto más posterior de la cara distal del primer molar superior (6d) y el plano vertical pterigoideo (Figura 16).

10. 6f-FH: altura dentoalveolar posterior superior; distancia del punto más bajo de la fisura media del primer molar superior (6f) al plano de Frankfurt (Figura 16).

11. Is-PMx: altura dentoalveolar anterior superior; distancia entre el borde incisal del incisivo central superior (Is) y el plano maxilar (PMx) (Figuras 17A y B).

12. Is-FH: altura dentomaxilar anterior superior; distancia entre el borde incisal del incisivo central superior (Is) y el plano de Frankfurt (Figura 18).

13. NB-FH: proyección anterior mandibular; ángulo formado por la intersección de la línea N-B y el plano de Frankfurt (Figura 19).

14. PMn-FH: inclinación del cuerpo mandibular; ángulo formado por la intersección del plano mandibular y el plano de Frankfurt. El plano mandibular se forma por la unión de los puntos Me (mentale), AGoR (antegonial derecho), AGoL (antegonial izquierdo) (Figura 20).

15. 1-PMn: angulación del incisivo inferior; ángulo formado por la intersección del eje axial del incisivo central inferior y el plano mandibular (Figura 21).

16. 1-NB: angulación clínica del incisivo inferior; ángulo formado por el eje axial del incisivo inferior y la línea NB (nasión-punto B) (Figura 22).

17. li-Me: altura dentoalveolar anterior inferior; distancia del borde incisal del incisivo inferior (li), a mentale (Me) (Figura 23).

18. 6f-PMn: altura dentoalveolar posterior inferior; distancia entre el punto más inferior de la fisura mesial del primer molar inferior al plano mandibular (medida perpendicular) (Figuras 24A y B).

19. AGo-D.

- AGoR-D: longitud del cuerpo mandibular derecho (Figura 25).

- AGoL-D: longitud del cuerpo mandibular izquierdo (Figura 25).

Distancia entre los puntos antegoniales derecho e izquierdo (AGoR) (AGoL) y el punto D (centro geométrico de la sínfisis.

20. BAR-BPR.

- BARR-BPRR: ancho de rama mandibular derecha (Figura 26).

- BARL-BPRL: ancho de rama mandibular izquierda (Figura 26).
Distancia entre los puntos de máxima concavidad del borde anterior (BAR) y posterior (BRP).

21. ES-AGo.

- ESR-AGoR: altura de rama derecha (Figura 27).

- ESL-AGoL: altura de rama izquierda (Figura 27).

Distancia entre el punto de mayor concavidad de la escotadura sigmoidea (ES) y el punto antegonial (punto más posterior y superior de la escotadura pregonial).

22. CdGo-VPt.

- CdGoR-VPt: inclinación de la rama mandibular derecha (Figura 28).

- CdGoL-VPt: inclinación de la rama mandibular izquierda.

Ángulo formado por la intersección de la línea Cd-Go (centro geométrico del cóndilo al punto más posterior e inferior del ángulo de la mandíbula) con el plano vertical.

23. < Go: ángulo goniaco; ángulo formado por la intersección del plano mandibular (Me-AGoR-

AGoL) y el plano posterior de la rama ascendente (ArR-ArL-GoR-GoL) (Figura 29).

24. Cd-PVt.

- CdR-PVt: ubicación anteroposterior de la ATM derecha.

- CdL-PVt: ubicación AP de la ATM izquierda.

Distancia entre centro geométrico de cóndilo $(\mathrm{Cd})$ al plano vertical pterigoideo (VPt) (Figura 30).

25. Cd-FH.

- CdR-FH: ubicación vertical de la ATM derecha.

- CdL-FH: ubicación vertical de la ATM izquierda. Distancia del centro geométrico del cóndilo $(\mathrm{Cd})$ a el plano de Frankfurt horizontal (FH) (Figura 30).

26. < ECd-FH.

- < ECdR-FH: ángulo del cóndilo derecho.

- < ECdL-FH: ángulo del cóndilo izquierdo.

Ángulo formado entre el eje condilar (Cd-punto medio de la porción inferior del cuello del cóndilo) y el plano de Frankfurt (Figura 31).

27. ECds-Ecdi.

- ECdsR-EcdiR: longitud condilar derecho.

- ECdsL-EcdiL: longitud condilar izquierdo.

Distancia entre el punto más alto del eje condilar y el punto más bajo del eje condilar situado en la intersección del eje condilar por una línea perpendicular a este eje, que pasa tangente al punto de mayor concavidad en la escotadura sigmoidea (Figura 32).

28. B-Me: altura del mentón; distancia entre el punto B (punto de mayor concavidad en la cara anterior del proceso dentoalveolar inferior) y el punto mentale (punto más inferior del reborde de la sínfisis) (Figura 33). 


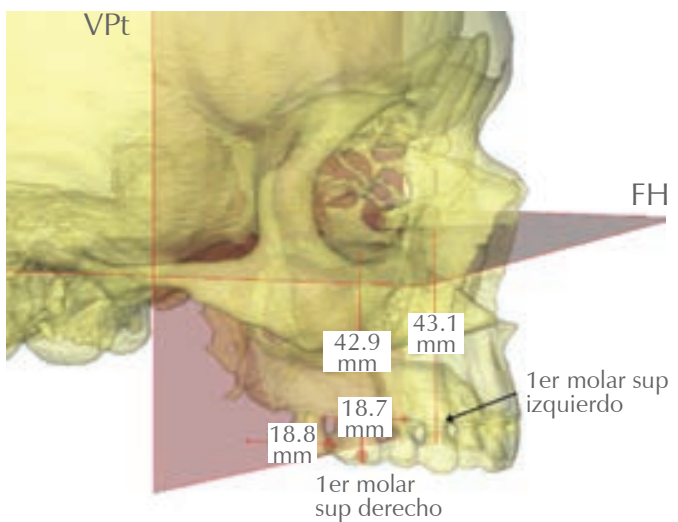

Figura 16: Ejemplo de medida 6d-VPt y 6f-FH de lado derecho e izquierdo. Autor: Trujillo. Norma: 6d-VPt; M: 14 mm/H: 16 mm. Aumento: mesialización del primer molar superior. Disminuido: distalización del primer molar superior. Norma: 6f- $\mathrm{FH} ; \mathrm{M}: 47 \mathrm{~mm} / \mathrm{H}: 51 \mathrm{~mm}$. Aumento: exceso de crecimiento vertical posterior sup. Disminuido: deficiencia de crecimiento vertical post. sup.

A

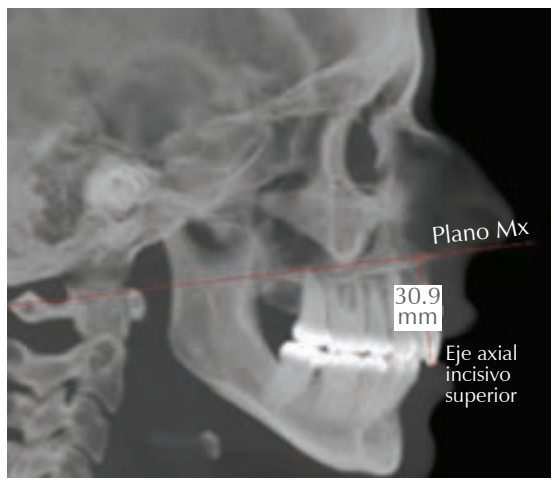

B

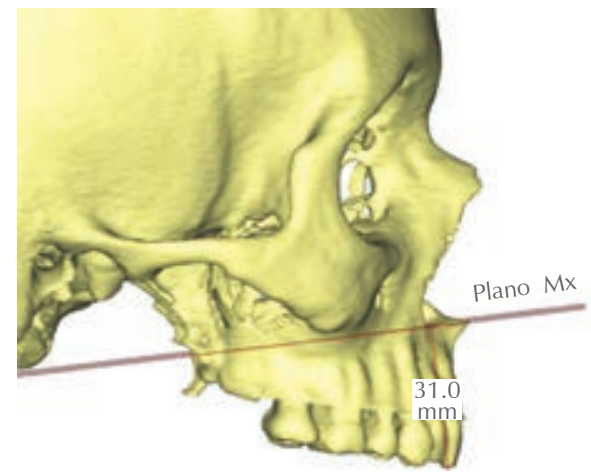

Figura 17: A) Ejemplo de medida Is-PMx. Imagen es la vista radiográfica, se observa el eje axial del incisivo superior que pasa por el borde incisal y el ápice de dicho órgano dentario. B) Ejemplo de medida Is-PMx, en este caso de $30.9 \mathrm{~mm}$. Autor: Trujillo. Norma clínica: $30 \pm 2 \mathrm{~mm}$. Aumento: exceso de crecimiento vertical dentoalveolar anterior sup. Disminuido: deficiencia de crecimiento vertical dentoalveolar anterior sup. $\mathrm{PMx}=$ plano maxilar formado por la unión de los puntos ENP y ENA.

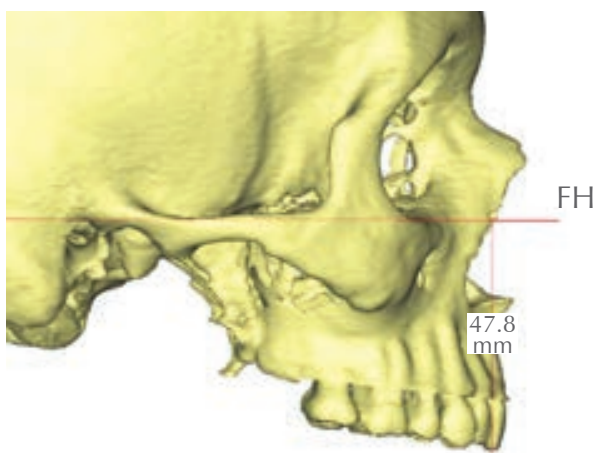

Figura 18: Ejemplo de medida Is-FH, en este caso de 47.8 mm. Autor: Trujillo. Norma clínica: $56 \pm 2 \mathrm{~mm}$. Aumento: exceso de crecimiento vertical anterior superior. Disminuido: deficiencia de crecimiento vertical anterior superior. $\mathrm{FH}=$ plano de Frankfurt.

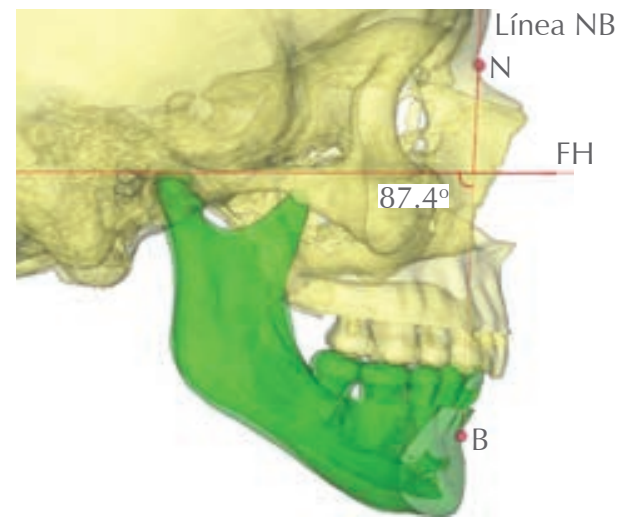

Figura 19: Ejemplo de medida Is- $\mathrm{FH}$, en este caso de $87.4^{\circ}$. Autor: Ricketts. Norma clínica: $88^{\circ} \pm 2^{\circ}$. Aumento: protrusión mandibular o prognatismo mandibular. Disminuido: retrusión mandibular o retrognatismo mandibular. $\mathrm{FH}=$ plano de Frankfurt.

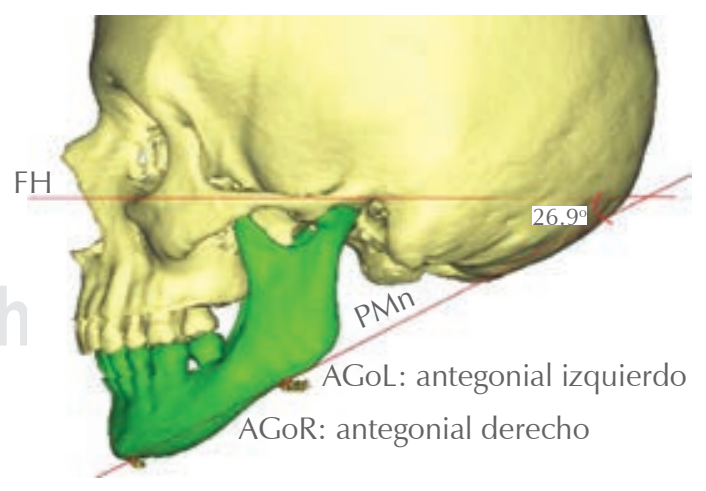

Figura 20: Ejemplo de medida PMn-FH, en este caso de 26.9o. Autor: Trujillo. Norma clínica: 22o \pm 8 o. Aumento: retroinclinación o posterorrotación del cuerpo mandibular. Disminuido: proinclinación o anterorrotación del cuerpo mandibular. $\mathrm{FH}=$ plano de Frankfurt, $\mathrm{PMn}=$ plano mandibular, $\mathrm{AGoR}=$ antegonial derecho, $\mathrm{AGoL}=$ antegonial izquierdo. 


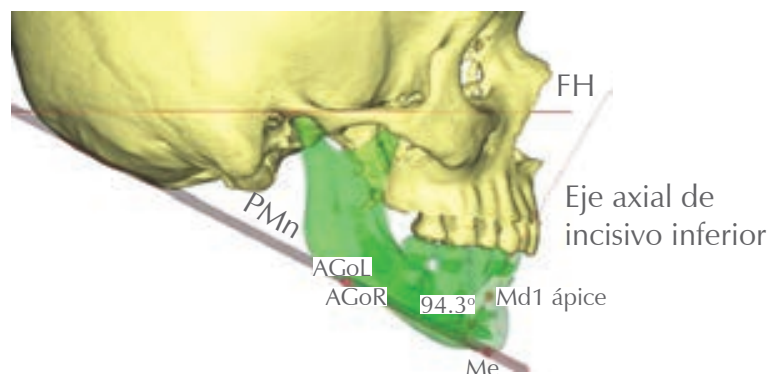

Figura 21: Ejemplo de medida 1-PMn, en este caso de 94.3․ Autor: Trujillo. Norma clínica: $91^{\circ} \pm 5^{\circ}$. Aumento: proinclinación o labioversición de los incisivos inferiores. Disminuido: retroinclinación o linguoversión de los incisivos inferiores.

$\mathrm{FH}=$ plano de Frankfurt, $\mathrm{PMn}=$ plano mandibular, $\mathrm{AGoR}=$ antegonial derecho, $\mathrm{AGoL}=$ antegonial izquierdo, $\mathrm{Md} 1=$ borde incisal incisivo inferior, Md1 ápice = ápice de incisivo inferior.

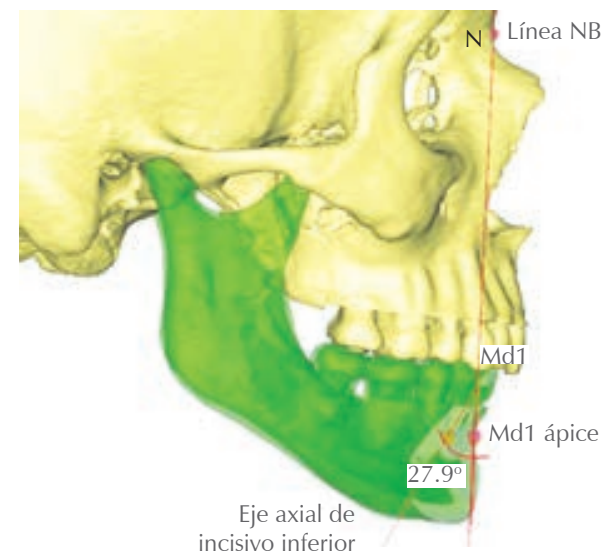

Figura 22: Ejemplo de medida 1-NB, en este caso de 27.9. Autor: Steiner. Norma clínica: $20^{\circ}$ o $4 \mathrm{~mm}\left( \pm 3^{\circ}\right)$. Aumento: labioversión clínica de los dientes anteriores inferiores. Disminuido: linguoversión clínica de los dientes anteriores inferiores. $\mathrm{N}=$ nasión, $\mathrm{B}=$ punto más cóncavo de la cara anterior del reborde alveolar inferior, $\mathrm{Md} 1=$ borde incisal incisivo inferior, $\mathrm{Md} 1$ ápice $=$ ápice de incisivo inferior.
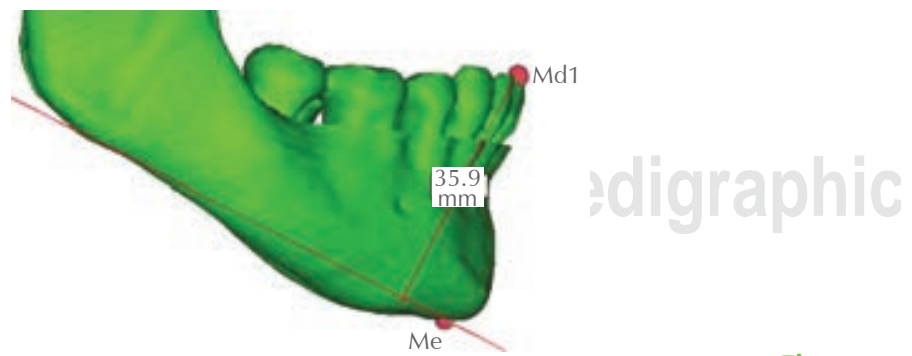

Figura 23: Ejemplo de medida li-Me, en este caso de 35.9 mm. Autor: Trujillo. Norma clínica: mujeres: 40 mm/hombres $44 \mathrm{~mm}$ ( $\pm 2 \mathrm{~mm}$ ). Aumento: exceso vertical dentoalveolar anterior inferior. Disminuido: deficiencia vertical dentoalveolar anterior inferior.

$\mathrm{Me}=$ mentale, $\mathrm{Md} 1=$ borde incisal de incisivo inferior.
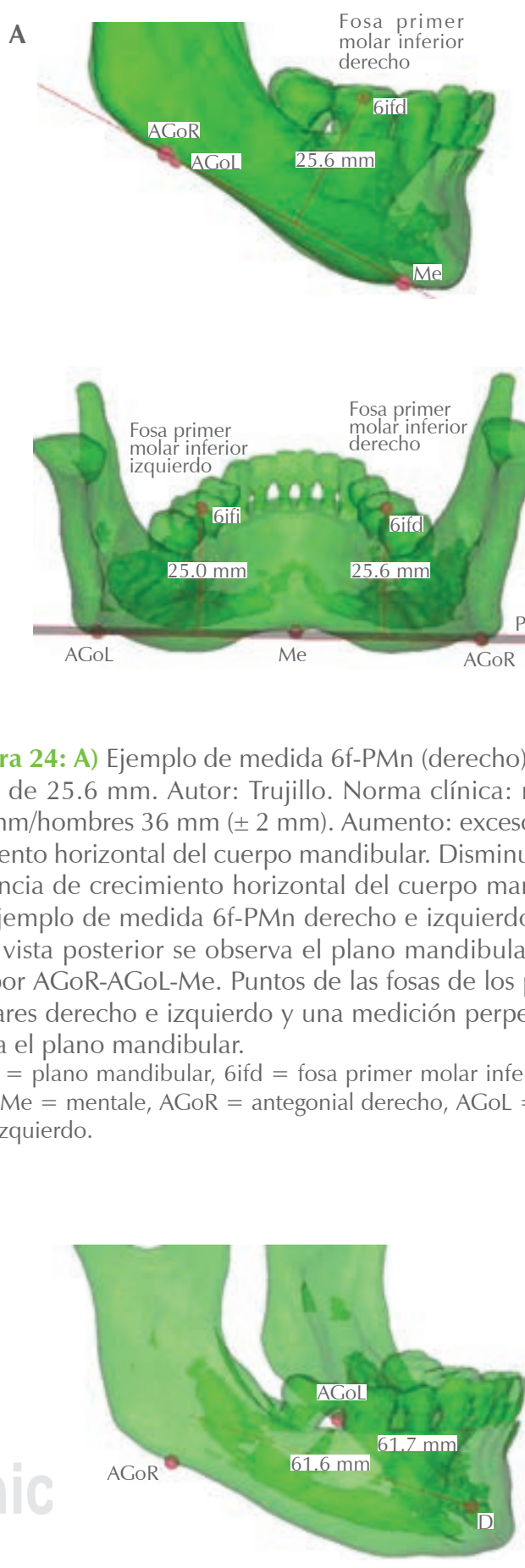

B

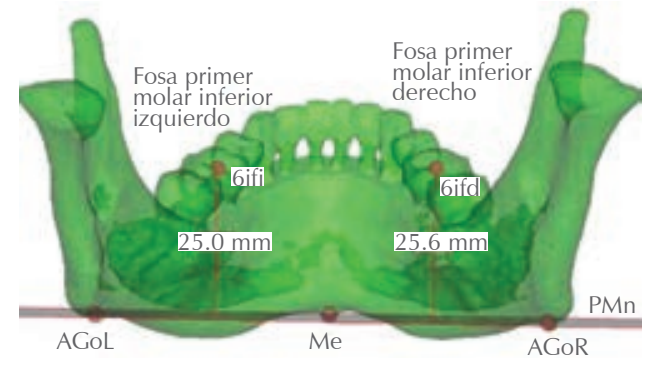

Figura 24: A) Ejemplo de medida 6f-PMn (derecho), en este caso de $25.6 \mathrm{~mm}$. Autor: Trujillo. Norma clínica: mujeres: $32 \mathrm{~mm} /$ hombres $36 \mathrm{~mm}$ ( $\pm 2 \mathrm{~mm}$ ). Aumento: exceso de crecimiento horizontal del cuerpo mandibular. Disminuido: deficiencia de crecimiento horizontal del cuerpo mandibular. B) Ejemplo de medida 6f-PMn derecho e izquierdo. Desde esta vista posterior se observa el plano mandibular formado por AGoR-AGoL-Me. Puntos de las fosas de los primeros molares derecho e izquierdo y una medición perpendicular hasta el plano mandibular.

$\mathrm{PMn}=$ plano mandibular, 6 ifd $=$ fosa primer molar inferior derecho, $\mathrm{Me}=$ mentale, $\mathrm{AGoR}=$ antegonial derecho, $\mathrm{AGoL}=$ antegonial izquierdo.

Figura 25: Ejemplo de medida AGoR-D: $61.6 \mathrm{~mm} / \mathrm{AGoL-D:}$ $61.7 \mathrm{~mm}$. Autor: Trujillo. Norma clínica: mujeres: $45 \mathrm{~mm} / \mathrm{hom}-$ bres $50 \mathrm{~mm}$ ( $\pm 2 \mathrm{~mm}$ ). Aumento: exceso de crecimiento horizontal o longitud del cuerpo mandibular. Disminuido: deficiencia de crecimiento horizontal o longitud del cuerpo mandibular. $\mathrm{D}=$ centro geométrico de la sínfisis, $\mathrm{AGoR}=$ antegonial derecho, $\mathrm{AGoL}=$ antegonial izquierdo. 


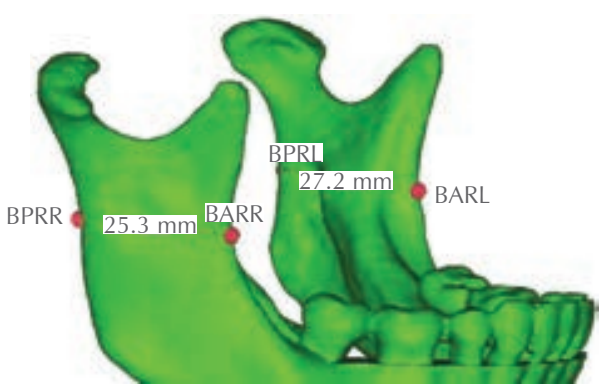

Figura 26: Ejemplo de medida BARR-BPRR: $25.3 \mathrm{~mm} / \mathrm{BARL}-$ BPRL: $27.2 \mathrm{~mm}$. Autor: Trujillo. Norma clínica: $30 \pm 3 \mathrm{~mm}$. Aumento: exceso de crecimiento horizontal o ancho de la rama mandibular. Disminuido: deficiencia de crecimiento horizontal o ancho de la rama mandibular.

$\mathrm{BARR}=$ borde anterior rama derecho, $\mathrm{BARL}=$ borde anterior rama izquierdo, $\mathrm{BPRR}=$ borde posterior de rama derecho, $\mathrm{BPRL}=$ borde posterior de rama izquierda.

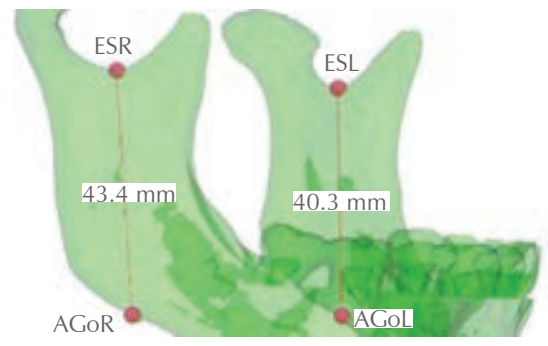

Figura 27: Ejemplo de medida ESR-AGoR: $43.4 \mathrm{~mm} / \mathrm{ESL}-$ AgoL: $40.3 \mathrm{~mm}$. Autor: Trujillo. Norma clínica: mujeres: $45 \mathrm{~mm} /$ hombres: $50 \mathrm{~mm}( \pm 3)$. Aumento: exceso de crecimiento vertical o aumento de longitud de rama ascendente mandibular. Disminuido: deficiencia de crecimiento vertical o aumento de longitud de rama ascendente mandibular. $\mathrm{ESR}=$ escotadura sigmoidea derecha, $\mathrm{ESL}=$ escotadura sigmoidea izquierda, $\mathrm{AGoR}=$ punto más posterior y superior de la escotadura pregonial derecha, $\mathrm{AGoL}=$ punto más posterior y superior de la escotadura pregonial izquierda.

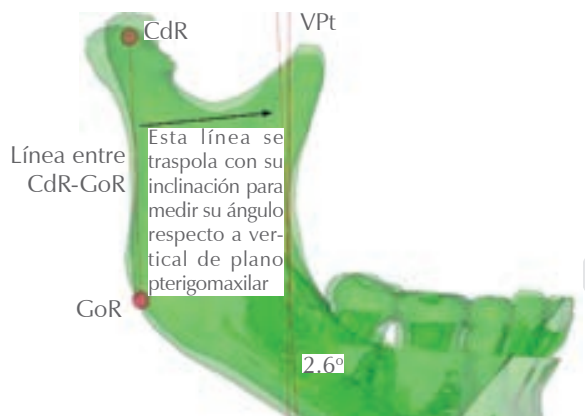

Figura 28: Ejemplo de medida CdGoR-VPt: 2.6 (ejemplo de lado derecho). Autor: Trujillo. Norma clínica: $+5^{\circ}\left( \pm 3^{\circ}\right)$. Aumento: anterorrotación o proinclinación de la rama ascendente. Disminuido: posterorrotación o retroinclinación de la rama ascendente.

$\mathrm{CdR}=$ centro de cóndilo derecho, GoR = gonión derecho, $\mathrm{VPt}=$ plano vertical pterigoideo.

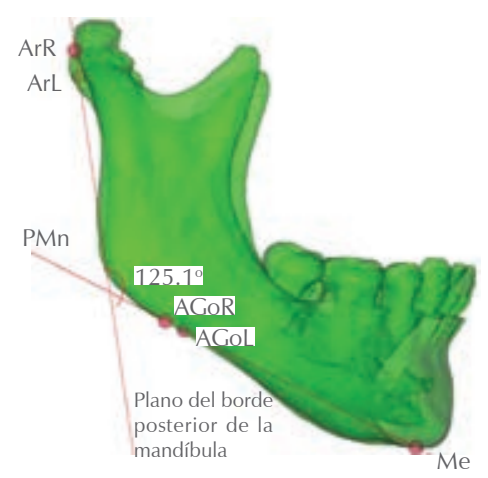

Figura 29: Ejemplo de medida del ángulo goniaco: $122.8^{\circ}$. Autor: Biorg. Norma clínica: $125^{\circ}\left( \pm 5^{\circ}\right)$. Aumento: hipergonia, ángulo goniaco abierto u obtuso. Disminuido: hipogonia, ángulo goniaco cerrado o agudo.

$\operatorname{ArR}=$ punto más posterior de cóndilo derecho, $\operatorname{ArL}=$ punto más posterior de cóndilo izquierdo.

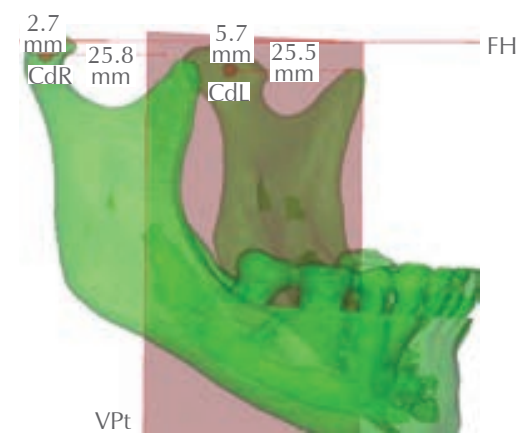

Figura 30: Ejemplo de medida de CdR a VPt (25.8 mm) y FH (2.7 mm). CdL a VPt (25.5 mm) y FH (5.7 mm). Autor: Trujillo. Norma: Cd-VPt 30 mm ( \pm 3 mm). Aumento: antero-posición de cóndilo y ATM = acortamiento de base craneal posterior. Disminuido: retroposición de cóndilo y ATM = aumento de longitud de base craneal posterior. Norma: Cd-FH 4 mm ( $\pm 1 \mathrm{~mm}$ ) (por debajo de plano FH). Aumento: ínfero-posición de cóndilo y ATM. Disminuido: supero-posición de cóndilo y ATM.

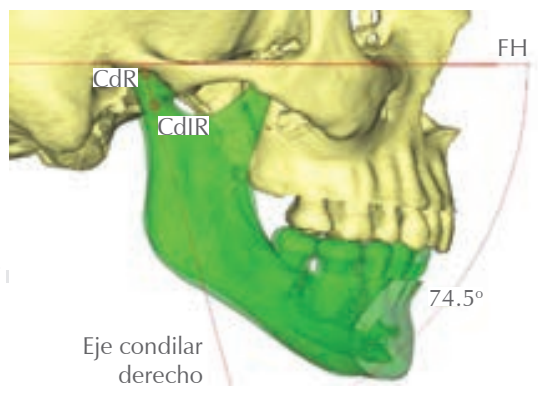

Figura 31: Ejemplo de medida < ECdR: $74.5^{\circ}$ (ejemplo de lado derecho). Autor: Trujillo. Norma clínica: $68^{\circ}\left( \pm 6^{\circ}\right)$. Aumento: postero-rotación del eje condilar o vector de crecimiento condilar. Disminuido: antero-rotación del eje condilar o vector de crecimiento condilar.

$\mathrm{CdR}=$ centro geométrico del cóndilo derecho, $\mathrm{CdIR}=$ condilar inferior: punto medio en la porción más angosta del cuello del cóndilo. 


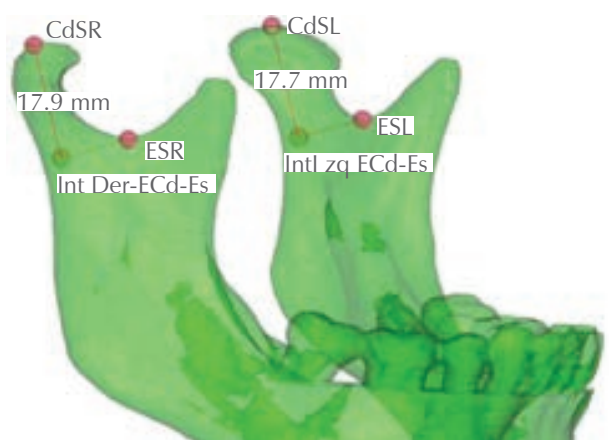

Figura 32: Ej. de medida ECdSR-ECdIR: $17.9 \mathrm{~mm} / \mathrm{ECdSI}-$ EcdIL: $17.7 \mathrm{~mm}$. Autor: Trujillo. Norma clínica: $24 \mathrm{~mm}( \pm 2$ $\mathrm{mm})$. Aumento: hiperplasia longitudinal condilar. Disminuido: hipoplasia longitudinal condilar.

$\mathrm{CdSR}=$ punto más alto en el eje condilar derecho, $\mathrm{CdSL}=$ punto más alto en el eje condilar izquierdo, ESR = punto más cóncavo de escotadura sigmoidea derecho, ESL = punto más cóncavo de escotadura sigmoidea izquierda, ECdID = punto de intersección de eje condilar y perpendicular que pasa por ESR, ECdII = punto de intersección de eje condilar y perpendicular que pasa por ESL.

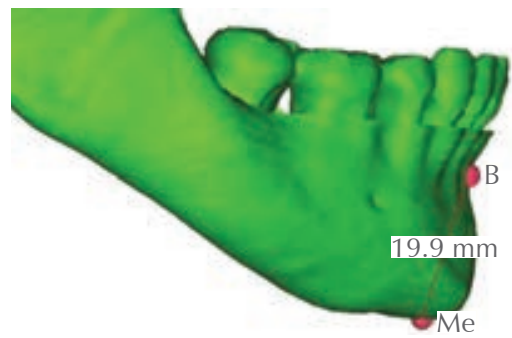

Figura 33: Ejemplo de medida B-Me, en este ejemplo es 19.9 mm. Autor: Trujillo. Norma clínica: 21 mm (desviación: 20-25 $\mathrm{mm})$. Aumento: exceso del crecimiento vertical del mentón. Disminuido: deficiencia del crecimiento vertical del mentón. $\mathrm{B}=$ punto de mayor concavidad de cara anterior del proceso dentoalveolar inferior, $\mathrm{Me}=$ punto más inferior del reborde de la sínfisis.

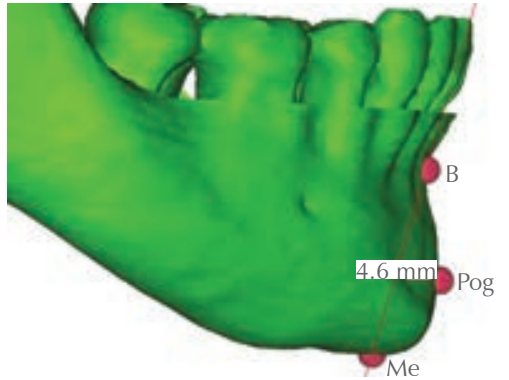

Figura 34: Ejemplo de medida BMe-Pog, en este ejemplo es $4.6 \mathrm{~mm}$. Autor: Trujillo. Norma clínica: $6 \mathrm{~mm}( \pm 1 \mathrm{~mm})$. Aumento: hiperplasia horizontal del mentón, macrogenia. Disminuido: hipoplasia horizontal del mentón, microgenia. $\mathrm{B}=$ punto de mayor concavidad de cara anterior del proceso dentoalveolar inferior, $\mathrm{Me}=$ punto más inferior del reborde de la sínfisis, Pog = punto más anterior de la sínfisis.

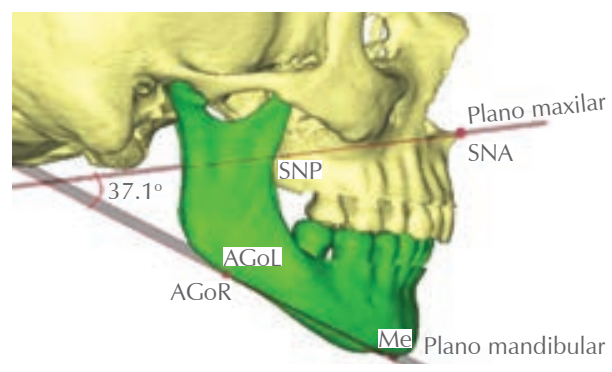

Figura 35: Ejemplo de medida de PMx-PMn, en este ejemplo $37.1^{\circ}$. Autor: Trujillo. Norma clínica: $25^{\circ}\left( \pm 10^{\circ}\right)$. Aumento: aumento de la relación vertical maxilo-mandibular. Disminuido: disminución de la relación vertical maxilo-mandibular. Plano mandibular: formado por AGoR-AGoL-Me, plano maxilar formado por SNA-SNP.

AGoR $=$ punto más posterior y superior de la escotadura pregonial derecha, $\mathrm{AGoL}=$ punto más posterior y superior de la escotadura pregonial izquierda, $\mathrm{Me}=$ punto más inferior de la sínfisis, $\mathrm{SNA}=$ espina nasal anterior, $\mathrm{SNP}=$ espina nasal posterior.

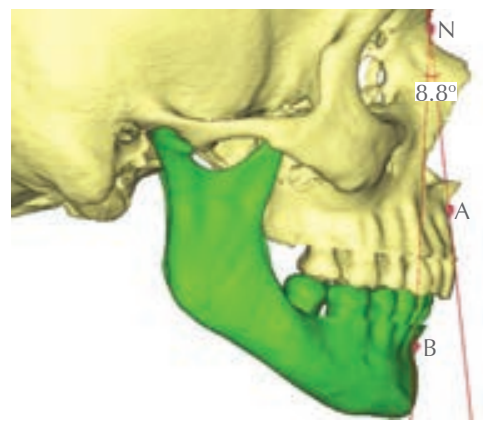

Figura 36: Ejemplo de medida de $<\mathrm{ANB}$, en este ejemplo

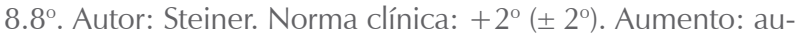
mento de la relación horizontal maxilo-mandibular. Disminuido: disminución de la relación horizontal maxilo-mandibular. $\mathrm{N}$ = nasión, $\mathrm{A}=$ punto de mayor concavidad en la cara anterior del reborde alveolar superior, $\mathrm{B}=$ punto de mayor concavidad de la cara anterior del reborde alveolar inferior.

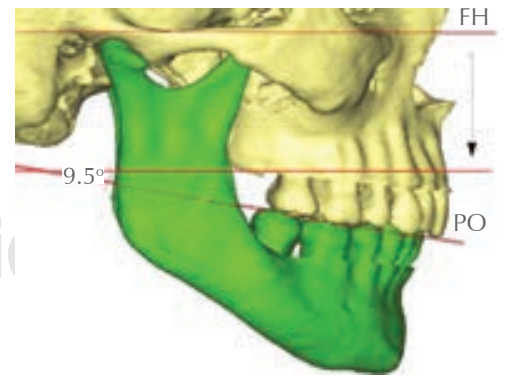

Figura 37: Ejemplo de medida PO-FH, en este ejemplo 9.5 . Autor: Wolford. Norma clínica: $-8^{\circ}( \pm 3)$. Aumento: posterorrotación o retroinclinación del plano oclusal. Disminuido: anterorrotación o proinclinación del plano oclusal. $\mathrm{FH}=$ plano de Frankfurt, $\mathrm{PO}=$ plano oclusal, flecha negra $=$ muestra el traslado del plano horizontal de $\mathrm{FH}$ para medir el ángulo que forma con el plano oclusal. 


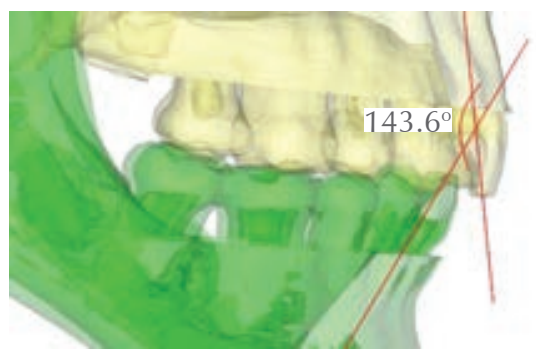

Figura 38: Ejemplo de medida 1/1 (ángulo interincisal), en este ejemplo de $143.6^{\circ}$. Autor: Riquetts. Norma clínica: $130^{\circ}\left( \pm 6^{\circ}\right)$. Aumento: aumento de la relación interincisiva. Disminuido: disminución de la relación interincisiva.

$\mathrm{FH}=$ plano de Frankfurt, $\mathrm{PO}=$ plano oclusal, flecha negra: muestra el traslado del plano horizontal de $\mathrm{FH}$ para medir el ángulo que forma con el plano oclusal.

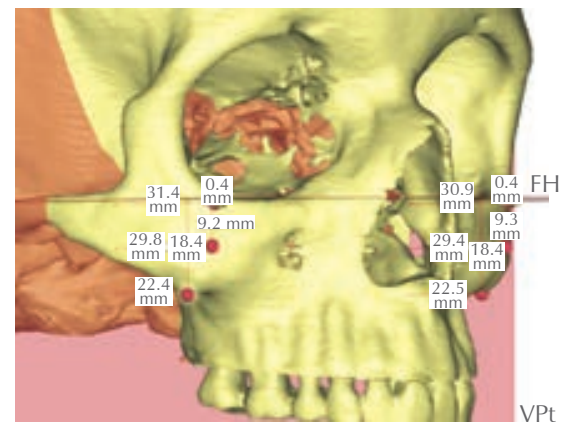

Figura 39: Ejemplo de las mediciones de las prominencias malar superior, media e inferior de lado derecho e izquierdo. Autor: Trujillo. Norma clínica: PMS-VPt: $41 \pm 3 \mathrm{~mm}$. Norma clínica: PMM-VPt: $39 \pm 3 \mathrm{~mm}$. Norma clínica: PMIVPt: 29 mm (27-34 mm). Aumento: antero-posición de la PMS, PMM o PMI. Disminuido: postero-posición de la PMS, PMM o PMI. Norma clínica: PMS-FH: 1 mm (0-2 mm). Norma clínica: PMM-FH: $14 \pm 1 \mathrm{~mm}$. Norma clínica: PMI-FH: $24 \pm 4$ mm. Aumento: infraposición de la PMS, PMM o PMI. Disminuido: supraposición de la PMS, PMM o PMI.

\section{A}

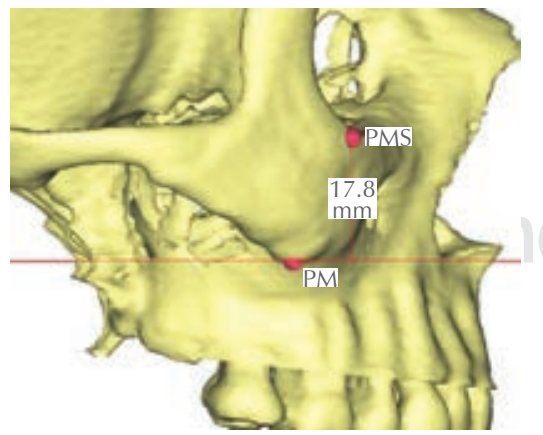

Figura 40: A) Ejemplo de medida PMS-PMI derecha: en este caso $17.8 \mathrm{~mm}$. Autor: Trujillo. Norma clínica: mujeres 21 mm/hombres: 23 ( $\pm 2 \mathrm{~mm}$ ). Aumento: exceso de dimensión vertical de tercio medio der. Disminuido: deficiencia de dimensión vertical de tercio medio der.

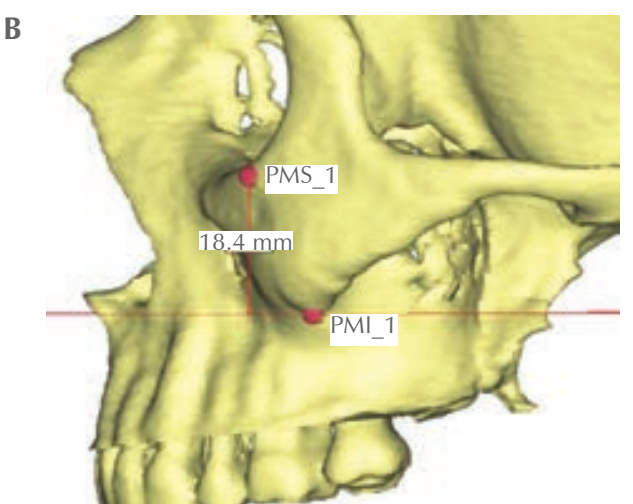

Figura 40: B) Ejemplo de medida PMS-PMI izquierdo: en este caso $18.4 \mathrm{~mm}$. Autor: Trujillo. Norma clínica: mujeres $21 \mathrm{~mm} /$ hombres: 23 ( $\pm 2 \mathrm{~mm}$ ). Aumento: exceso de dimensión vertical de tercio medio der. Disminuido: deficiencia de dimensión vertical de tercio medio der.

A

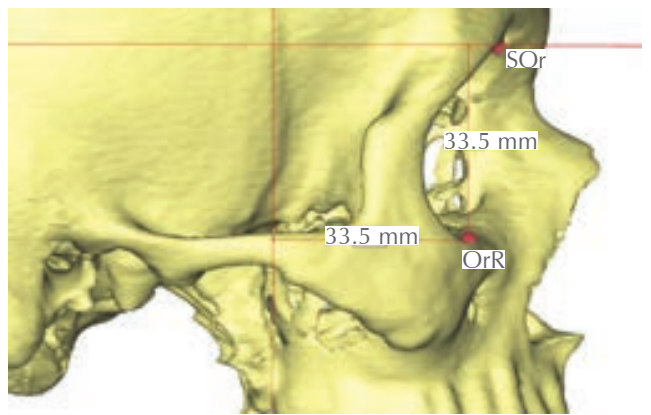

B

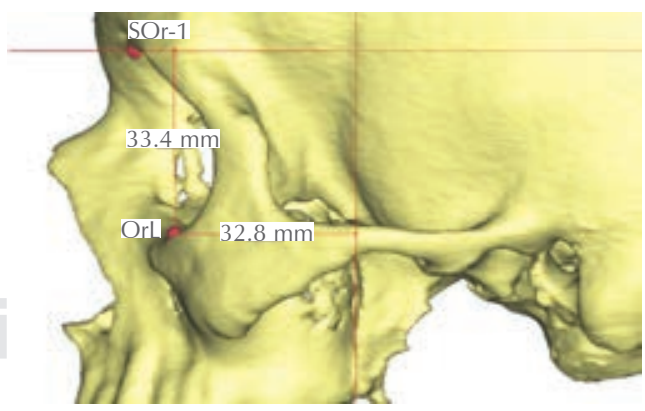

Figura 41: A) Ejemplo de medida Or-VPt derecha y Or-SOr derecha: en este caso 33.5 y $33.5 \mathrm{~mm}$ respectivamente. Autor: Trujillo. Norma clínica: Or-VPt: $36 \pm 3 \mathrm{~mm}$. Norma clínica: Or-SOr: $37 \pm 3 \mathrm{~mm}$. B) Ejemplo de medida Or-VPt izquierda y Or-SOr izquierda: en este caso 32.8 y $33.4 \mathrm{~mm}$ respectivamente. Autor: Trujillo. Norma clínica: Or-VPt: 36 \pm 3 mm. Norma clínica: Or-SOr: $37 \pm 3 \mathrm{~mm}$. 
29. BMe-Pog: proyección del mentón; distancia entre la línea B-Me y el punto más anterior de la sínfisis (Pog). Medida perpendicular a la línea B-Me (Figura 34).

30. PMx-PMn: relación vertical maxilomandibular; ángulo formado por la intersección de los planos mandibular y el plano maxilar (Figura 35).

31. < ANB: relación horizontal intermaxilar; ángulo formado por la intersección de la línea N-A y

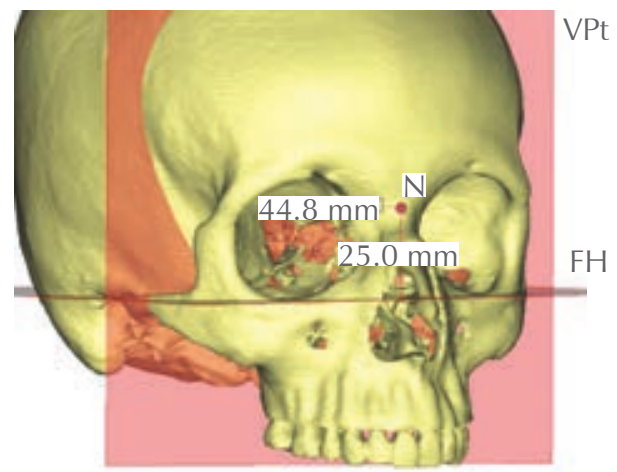

Figura 42: Ejemplo de las mediciones de N-VPt en este caso: $44.8 \mathrm{~mm}$ y la medida de $\mathrm{N}-\mathrm{FH}$ en este caso de $25.0 \mathrm{~mm}$. Autor: Trujillo. Norma clínica N-VPt: $50 \pm 3 \mathrm{~mm}$. Aumento: protrusión de la porción nasal. Disminuido: retrusión de la porción nasal. Autor: Trujillo. Norma clínica N-FH: $29 \pm 4$ $\mathrm{mm}$. Aumento: aumento de la dimensión vertical del tercio medio. Disminuido: disminución de la dimensión vertical del tercio medio.

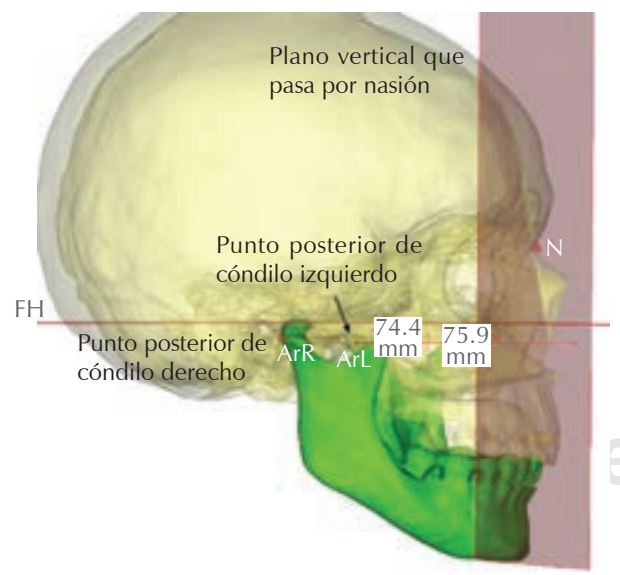

Figura 43: Ejemplo de medida de la profundidad facial de lado derecho y del lado izquierdo, en este caso el lado derecho es $74.4 \mathrm{~mm}$ y el lado izquierdo es $75.9 \mathrm{~mm}$. Autor: Trujillo. Norma clínica: mujeres: $84 \pm 3 \mathrm{~mm}$. Hombres: $91 \pm 3 \mathrm{~mm}$. Aumento: exceso de la dimensión horizontal facial o aumento de la profundidad facial. Disminuido: disminución de la dimensión horizontal facial o disminución de la profundidad facial.

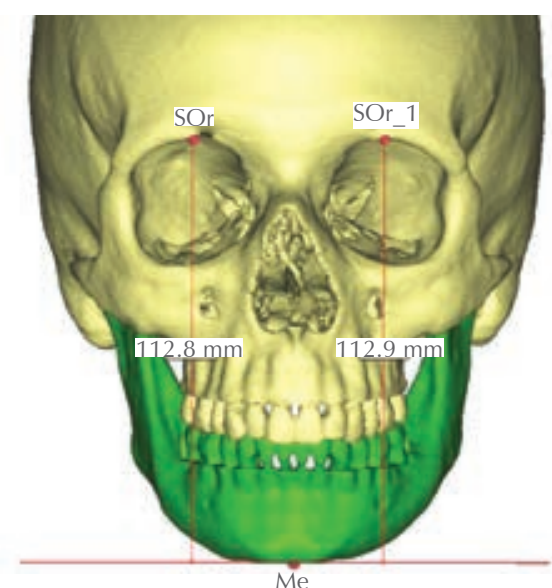

Figura 44: Ejemplo de medida de la altura facial de lado derecho e izquierdo: en este caso lado derecho $112.8 \mathrm{~mm}$ y lado izquierdo $112.9 \mathrm{~mm}$. Autor: Trujillo. Norma clínica: $\mathrm{CdP}^{\prime \prime}-\mathrm{N}^{\prime \prime} \times$ 1.618. Norma clínica: mujeres: $135 \mathrm{~mm}$. Hombres: $147 \mathrm{~mm}$. Aumento: exceso de crecimiento vertical facial, aumento de la altura facial. Disminuido: deficiencia de crecimiento vertical facial, disminución de la altura facial.

la línea N-B. Relaciona los dos maxilares entre si tomando como punto de conexión al punto nasión (Figura 36).

32. PO-FH: ángulo del plano oclusal del maxilar superior; ángulo formado por la intersección del plano oclusal (plano determinado por el borde de los incisivos superiores y las cúspides mesiovestibulares de los primeros molares superiores) y el plano de Frankfurt (Figura 37).

33. 1/1: ángulo interincisivo; ángulo formado por la intersección de los ejes axiales del incisivo superior e incisivo inferior (Figura 38).

34. PMS-VPt: proyección anteroposterior de la prominencia malar superior (PMS); distancia de la PMS a la vertical pterigoidea (VPt) (Figura 39).

35. PMS-FH: ubicación vertical de la prominencia malar superior (PMS); distancia de la PMS al plano horizontal de Frankfurt (FH) (Figura 39).

36. PMM-VPt: proyección antero-posterior de la prominencia malar media (PMM); distancia de la PMM a la vertical pterigoidea (VPt) (Figura 39).

37. PMM-FH: ubicación vertical de la prominencia malar media (PMM); distancia entre la PMM y el plano horizontal de Frankfurt (FH) (Figura 39).

38. PMI-VPt: proyección anteroposterior de la prominencia malar inferior (PMI); distancia de la PMI a la vertical pterigoidea (VPt) (Figura 39). 
39. PMI-FH: ubicación vertical de la prominencia malar inferior (PMI); distancia de la PMI al plano horizontal de Frankfurt (FH) (Figura 39).

40. PMS-PMI: dimensión vertical malar; distancia entre los puntos de la prominencia malar superior (PMS) y el de la prominencia malar inferior (PMI) (Figuras 40A y B).

41. Or-VPt: proyección del reborde infraorbitario; distancia del punto más inferior del reborde orbitario inferior (Or) al plano vertical pterigoideo (VPt) (Figuras $41 A$ y $B$ ).

42. Or-SOr: dimensión vertical de la órbita; distancia entre el punto Or y el punto más superior del reborde supraorbitario (SOr).

43. N-VPt: proyección anteroposterior de nasión (N); distancia entre el punto $\mathrm{N}$ y el plano vertical pterigoideo (VPt) (Figura 42).

44. N-FH: ubicación vertical de nasión (N); distancia entre el punto $\mathrm{N}$ y el plano horizontal de Frankfurt (Figura 42).

45. CdP"-N": profundidad facial; distancia entre los puntos condilar posterior ( $\mathrm{Ar}$ ) y nasión $(\mathrm{N})$ (Figura 43).

46. Sor"-Me": altura facial; distancia entre el punto más superior del reborde supraorbitario (SOr) y el punto más inferior de la sínfisis (Me) (Figura 44).

\section{CONCLUSIONES}

El análisis de tejidos duros de Trujillo cuenta con mediciones de todas las estructuras óseas involucradas en la cirugía ortognática, por lo que nos parece el análisis indispensable para el complemento diagnóstico al examen clínico, indispensable como análisis cefalométrico prequirúrgico.

En su aplicación a la planeación quirúrgica tridimensional este análisis es reproducible, es factible, es funcional, es operacional y aporta más conocimiento geométrico del paciente que otros análisis.

Por último, como las normas clínicas de este análisis fueron obtenidas de análisis bidimensionales, el equipo que realizó esta adaptación está trabajando en un estudio para obtener normas clínicas de este análisis en tres dimensionales.

\section{BIBLIOGRAFÍA}

1. Broadbent BS. A new X-ray technique and its application to orthodontia. Angle Orthod. 1931; 1: 45-66.

2. van Vlijmen OJ, Maal T, Bergé SJ, Bronkhorst EM, Katsaros C, Kuijpers-Jagtman AM. A comparison between 2D and 3D cephalometry on CBCT scans of human skulls. Int J Oral Maxillofac Surg. 2010; 39 (2): 156-160.
3. Xia JJ, Gateno J, Teichgraeber JF, Yuan P, Li J, Chen KC et al. Algorithm for planning a double-jaw orthognathic surgery using a computer-aided surgical simulation (CASS) protocol. Part 2: threedimensional cephalometry. Int J Oral Maxillofac Surg. 2015; 44 (12): $1441-1450$.

4. Smektała T, Jędrzejewski M, Szyndel J, Sporniak-Tutak K, Olszewski R. Experimental and clinical assessment of threedimensional cephalometry: a systematic review. J Craniomaxillofac Surg. 2014; 42 (8): 1795-1801.

5. Swennen GR, Schutyser F. Three-dimensional cephalometry: spiral multi-slice vs cone-beam computed tomography. Am J Orthod Dentofacial Orthop. 2006; 130 (3): 410-416.

6. Gateno J, Xia JJ, Teichgraeber JF. Effect of facial asymmetry on 2-dimensional and 3-dimensional cephalometric measurements. J Oral Maxillofac Surg. 2011; 69 (3): 655-662.

7. Anas IY, Bamgbose BO, Nuhu S. A comparison between $2 \mathrm{D}$ and $3 \mathrm{D}$ methods of quantifying facial morphology. Heliyon. 2019; 5 (6): e01880.

8. Gubler M, Ludlow JB, Mol A, Cevidanes L. Precision of cephalometric landmark identification: 3D vs. 2D. Oral Surg Oral Med Oral Pathol Oral Radiol. 2009; 107: 4-10.

9. Gui H, Wu J, Shen SG, Bautista JS, Voss PJ, Zhang S. Navigation-guided lateral gap arthroplasty as the treatment of temporomandibular joint ankylosis. J Oral Maxillofac Surg. 2014; 72 (1): 128-138.

10. Yitschaky O, Redlich M, Abed Y, Faerman M, Casap N, Hiller N. Comparison of common hard tissue cephalometric measurements between computed tomography $3 \mathrm{D}$ reconstruction and conventional 2D cephalometric images. Angle Orthod. 2011; 81 (1): 11-16.

11. Naini FB, Gill DS. Orthognathic surgery. principles, planning and practice. United Kingdom: John Wiley \& Sons Press; 2017.

12. Alexander DC, Koeberlein GM. Geometría. 5a ed. México: Cengage Learning Press; 2013.

13. Lehmann CH. Geometría Analítica. 3a ed. México: Limusa Press; 1999.

14. Madsen DP, Sampson WJ, Townsend GC. Craniofacial reference plane variation and natural head position. Eur J Orthod. 2008; 30 (5): 532-540.

15. Gateno J, Xia JJ, Teichgraeber JF. New 3-dimensional cephalometric analysis for orthognathic surgery. J Oral Maxillofac Surg. 2011; 69 (3): 606-622.

16. Bell WH, Jacobs JD, Quejada JG. Simultaneous repositioning of the maxilla, mandible, and chin. Treatment planning and analysis of soft tissues. Am J Orthod. 1986; 89 (1): 28-50.

17. Heufelder M, Wilde F, Pietzka S, Mascha F, Winter K, Schramm A et al. Clinical accuracy of waferless maxillary positioning using customized surgical guides and patient specific osteosynthesis in bimaxillary orthognathic surgery. J Craniomaxillofac Surg. 2017; 45 (9): 1578-1585.

18. Moorrees CF, Kean MR. Natural head position, a basic consideration in the interpretation of cephalometric radiographs. Am J Phys Anthrop. 1958; 16: 213-234.

19. Lundström $F$, Lundström $A$. Natural head position as a basis for cephalometric analysis. Am J Orthod Dentofacial Orthop. 1992; 101 (3): 244-247.

20. Damstra J, Fourie Z, Ren Y. Simple technique to achieve a natural position of the head for cone beam computed tomography. $\mathrm{Br} \mathrm{J}$ Oral Maxillofac Surg. 2010; 48 (3): 236-238.

21. Schatz EC, Xia JJ, Gateno J, English JD, Teichgraeber JF, Garrett FA. Development of a technique for recording and transferring natural head position in 3 dimensions. J Craniofac Surg. 2010; 21 (5): 1452-1455.

22. Zamora Montes de Oca CE. Compendio de Cefalometría. 2a ed. México: Amolca; 2010.

Conflicto de intereses: no hay conflicto de intereses. 